\title{
Quantitative Analysis of Scanning Force Microscopy Images of Nickel-Based Superalloys
}

\author{
Alain Hazotte $\left({ }^{1}\right)$, Michel Troyon $\left({ }^{2}\right)$ and Abderrahim Bourhettar $(2, *)$ \\ ( $\left.{ }^{1}\right)$ Laboratoire de Science et Génie des Matériaux Métalliques, URA CNRS 159, École des Mines, \\ Parc de Saurupt, 54042 Nancy Cedex, France \\ $\left({ }^{2}\right)$ Laboratoire de Microscopies Électronique et Tunnel, Université de Reims Champagne-Ardenne, \\ 21 rue Clément Ader, 51685 Reims Cedex 2, France
}

\begin{abstract}
Résumé. - Cet article présente des travaux récents visant à tester le potentiel de la microscopie à force atomique pour l'observation et l'analyse quantitative de la microstructure des superalliages monocristallins à base de nickel. Des images de résolution et contraste satisfaisants peuvent être obtenues en mode "topographique" (nanorugosimétrie de surfaces polies/attaquées) et en mode "mécanique" (contraste dû à une différence de rigidité élastique entre phases). Néanmoins, des effets associés à la taille non négligeable de la pointe utilisée introduisent des biais de mesure qui doivent être pris en compte. Différentes façons de les minimiser sont proposées et commentées.
\end{abstract}

\begin{abstract}
This paper presents very recent works on the use of scanning force microscopy (SFM/AFM) to image and quantify the microstructure of single-crystal nickel-based superalloys. Images with satisfying contrast and resolution can be obtained by working in either "topographic" mode (nanoprofilometry of polished/etched surfaces) or "mechanical" mode (contrast due to the difference of elastic stiffness between phases). Nevertheless, measurement bias results from the non-negligeable size of the probe tip. Some ways to minimize them are proposed and discussed.
\end{abstract}

\section{Introduction}

The continuous improvement of the mechanical properties of bulk materials requires to optimize their microstructure at more and more fine levels. This evolution towards nanometer scale opens new questions in terms of imaging and quantitative analysis. In that field, works have to be done to test the potentiality of new imaging techniques such as near-field microscopies [1], without to omit to examine carefully the different sources of errors which could hinder any quantitative exploitation of the necessarily attractive new images produced.

$\left.{ }^{*}\right)$ Now at: Centre de Microscopie Électronique, École Nationale Supérieure d'Électricité et de Mécanique, Université Hassan II - Oasis, Casablanca, Maroc 
a)

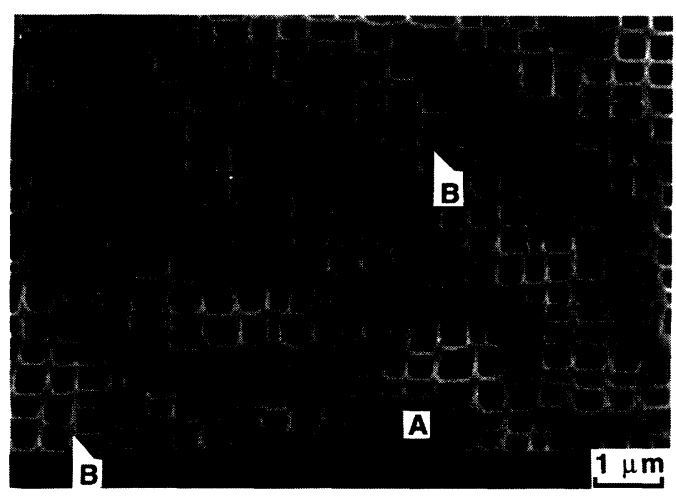

b)

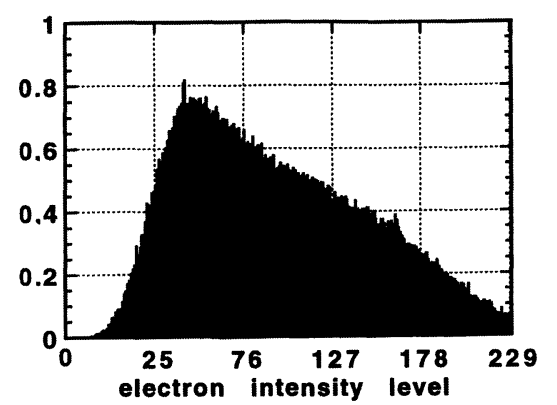

Fig. 1. - a) Typical microstructure of a single crystal of MC2 Ni-based alloy imaged by scanning electron microscopy in secondary electron mode (the surface is parallel to a $\{001\}$ crystallographic plane). b) Corresponding grey-level (electron-intensity) histogram.

This type of study was performed in the case of nickel-based alloys used to manufacture singlegrain high-temperature turbine components, i.e. the so-called "single-crystal superalloys" [2, 3]. These materials are strengthened by precipitation of hard and brittle sub-micronic particles - the $\gamma^{\prime}$ phase - in a more ductile matrix - the $\gamma$ phase -, which results in a rather simple microstructure illustrated in Figure 1a. Their high temperature strength and stability is mainly correlated with the $\gamma^{\prime}$ precipitate volume fraction, size, shape and spatial arrangement [4]. Scanning electron microscopy (SEM) is the most widely used imaging technique for superalloys ( $c f$. Fig. 1). $A$ priori, it seems to be suitable for their quantitative characterization, with regard to the good visual quality of the images obtained (Fig. 1a) and to the possibility to scan large surfaces in reasonable time. It has been effectively largely used to evidence relative microstructure changes, but it actually fails in providing absolute quantitative values of relevant structural parameters [4]. Indeed, as $\gamma$ and $\gamma^{\prime}$ phases have relatively close chemical compositions, the sample surface has to be etched then observed in secondary electron mode, which results in some bias due to the non linearity of the secondary electron intensity as a function of the surface topography $[4,5]$. For instance, large plateaux of matrix (zones A in Fig. 1a) are characterized by a non-homogeneous intensity level, while thin matrix channels (zones B in Fig. 1a) are artificially enlarged due to the over-intensity associated with steep steps on the surface. The difficulty of correctly discriminating $\gamma$ and $\gamma^{\prime}$ phases is evident when looking at intensity-level histograms associated with SEM images (cf. Fig. 1b).

Near-field microscopies - i.e. scanning tunnelling microscopy (STM), atomic force microscopy (AFM), scanning force microscopy (SFM), etc ... ( $\left.{ }^{1}\right)$ - were initially developed to investigate structures at the atomic scale [6,7]. However, they can also be used to observe surface areas of some $\mu \mathrm{m}^{2}$, the main technical limitation in this field being the non-linearity of piezo-electric components. As the time for image acquisition is now becoming reasonable, they are $a$ priori suitable for systematic observation of polyphase materials such as industrial metallic alloys. Our first attempt to image the microstructure of single-crystal superalloys by such techniques focused

\footnotetext{
(1) Initially, atomic force microscopy (AFM) was designating the near-field microscopy technique in which the information measured and imaged is the repulsive force between the atoms of the observed surface and those of the tip. Then, the term was extended to other modes of observation developed on the same principle but with different origins for the force on the tip (contact, friction, magnetic, ...). Now it is recommended to use scanning force microscopy (SFM) to name these techniques, AFM recovering its initial meaning.
} 




Fig. 2. - Schematic working principle of the SFM developed in the Laboratoire de Microscopies Electronique et Tunnel, Reims (see Ref. [14] for more details).

on STM [8]. Unfortunately, we failed in providing non-noisy images, which was attributed to the presence of a passivation layer on the sample surface. SFM gave much better results [9-12], and the aim of this paper is to summarize them. Firstly, the principle of SFM and the different imaging modes tested will be briefly presented. Then, the main inherent sources of measurement bias will be discussed and different ways to limitate them or to take them into account will be proposed.

\section{SFM Imaging of Nickel-Based Superalloys}

2.1 General Experimental Procedure. - SFM observations were performed using either a commercial microscope (Nanoscope II of Digital Instrument) or a microscope developed in the Laboratoire de Microscopies Electronique et Tunnel [13, 14]. The latter one, the principle of which is schematized in Figure 2, was designed to be inserted in and combined with a scanning electron microscope, in order to take advantage of the complementarity of the two techniques. The sample displacement along $x, y$ (parallel to the sample surface) and $z$ (perpendicular) axes is controlled through a piezo-electric ceramic tube. In our case, the $x-y$ scanning area was typically about $10 \times 10 \mu \mathrm{m}^{2}$ wide, while the $z$ dynamic range was about $\pm 1.5 \mu \mathrm{m}$. The tip probe used was a $\mathrm{Si}_{3} \mathrm{~N}_{4}$ single crystal attached to a cantilever the displacement of which is detected and controlled through an optical device. In the device schematized in Figure 2, the four segment photodiode enables to follow not only the cantilever displacement along $z$ direction, but also 


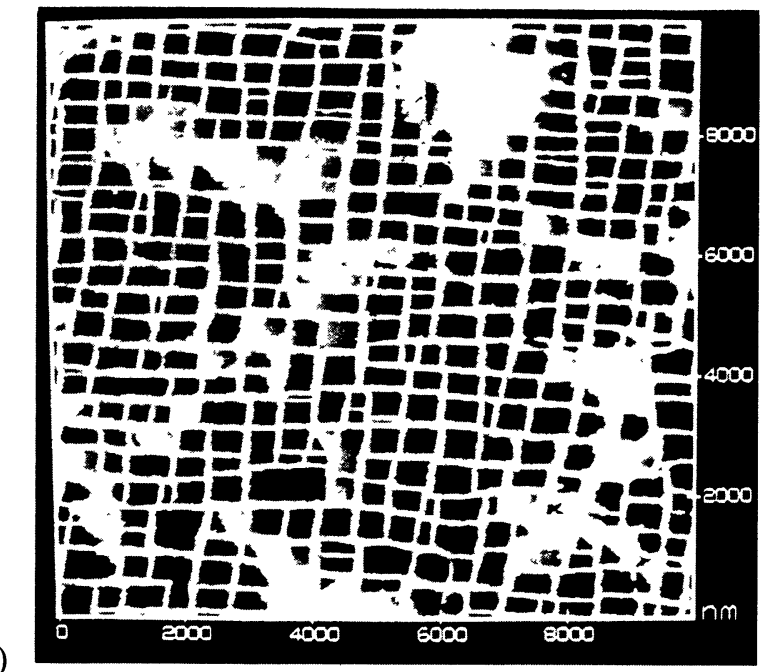

a)

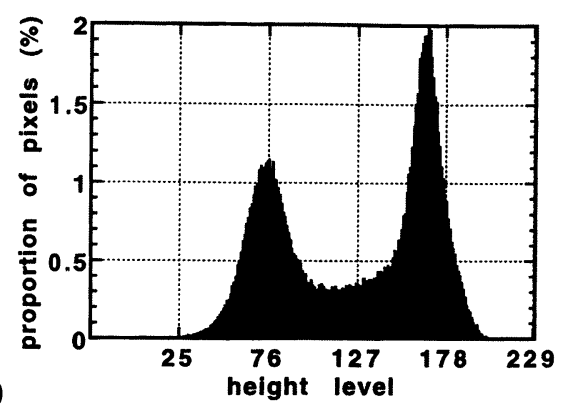

Fig. 3. - a) Microstructure of MC2 alloy imaged by SFM in topographic mode. b) Corresponding heightlevel histogram.

its torsion resulting from lateral frictional forces (tribological mode). This function has not been used in our case.

The samples observed were cut in single crystals of an industrial nickel-based superalloy denoted MC2 [15]. Their main surfaces were always parallel to a $\{001\}$ plane of the crystallographic lattice common to both $\gamma$ and $\gamma^{\prime}$ phases (same orientation as for Fig. 1). They were mechanically polished with grit disks then diamond paste down to $0.1 \mu \mathrm{m}$. Then, the subsequent preparation depended on the type of imaging mode used.

\subsection{Different Imaging Modes.}

2.2.1 "Chemical" Images. - $\gamma$ and $\gamma^{\prime}$ phases have not the same chemical compositions and crystallographic structures ( $\gamma^{\prime}$ phase has an f.c.c., $\mathrm{Ni}_{3} \mathrm{Al}$-based, ordered structure while $\gamma$ phase has an f.c.c., Ni-based, disordered structure). Thus, it is theoretically possible to get a contrast between phases on perfectly flat surfaces if working in classical atomic force mode. We made just a few attempts in this direction, without encouraging results. This was in fact not unexpected with regard to our first experience in tunnelling microscopy which led us to suspect the presence of a passivation layer on the sample surface. Moreover, it is unrealistic to aim at a surface preparation sufficiently good to scan large surface areas while conserving a $z$-resolution sufficiently low to contrast the phases.

2.2.2 Topographic Images. - Until now, our best images have been obtained by scanning preetched surfaces and controlling the $z$-displacement to hold the cantilever top at a constant height. In this mode, SFM is actually working as a nanoprofilometer, providing an image of the topography of the etched surface. The sample preparation was the same as for SEM observation, i.e. mechanical polishing then immersion in an acid solution, although the etching time/depth can be smaller. Figure $3 \mathrm{a}$ presents an example of the images obtained. Their resolution is comparable 


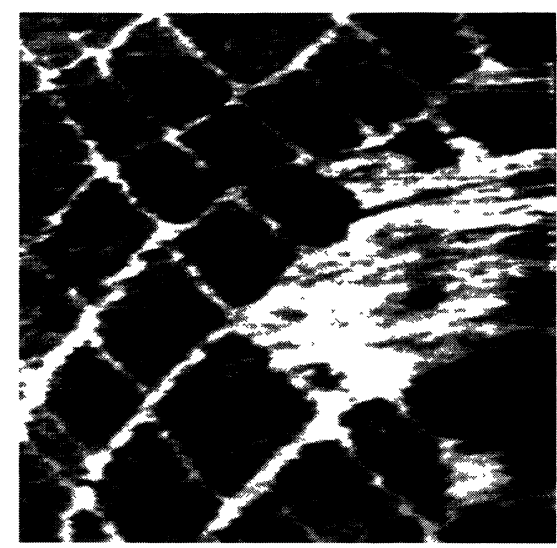

Fig. 4. - Microstructure of MC2 alloy imaged by SFM in "mechanical” mode (image size $=3.2 \times 3.2 \mu \mathrm{m}$ ).

to the one of SEM images, although their visual effect is somewhat not so attractive. But the important point is that the height level of each phase is much more homogeneous than its intensity level in SEM images. Consequently, the associated height level histograms are clearly bimodal, as illustrated in Figure $3 \mathrm{~b}$. This a priori enables an easy subsequent binarisation of the images.

2.2.3 "Mechanical" Images. - Very recently, a novel working mode called "Scanning LocalAcceleration Microscopy" (SLAM) was also tested. In that case, the sample is periodically moved up and down ( $z$ wobble), using a small piezo-electric disk added to the piezo-scanner ( $c f$. Fig. 2). One follows the oscillatory response of the cantilever, which amplitude and phase are directly dependent on the elasticity and viscosity of the material, respectively. The theory of this regime has been developed by Burham et al. [16]. Their analysis shows that modulating the position of the sample at frequencies above the highest system resonance gives the clearest difference in cantilever response for the variations in elastic modulus of stiff samples, whereas the low frequency regime is well adapted to much more compliant materials such as biological ones [17, 18]. Working at high excitation frequencies $(>100 \mathrm{kHz})$, we were able to obtain a satisfying contrast between $\gamma$ and $\gamma^{\prime}$ metallic phases even though their Young moduli are only slightly different. This result is illustrated by the image in Figure 4, in which $\gamma$ phase (Young modulus of about $130 \mathrm{GPa}$ ) appears in bright contrast and $\gamma^{\prime}$ phase (Young modulus of about $115 \mathrm{GPa}$ ) in dark. As far as we know, it is the first time that SFM can be used in "SLAM" working mode to image metallic phases with different Young moduli. Moreover, the ratio of the mean amplitudes measured for $\gamma$ and $\gamma^{\prime}$ phases is rather close to the ratio of their moduli. This working mode has therefore a great potential interest, not only to image structures, but particularly to estimate the stiffness of in-situ very small particles.

\section{Quantitative Analysis of SFM Images}

The volume fraction of $\gamma$ and $\gamma^{\prime}$ phases was measured from SFM images acquired in the topographic mode $[11,12]$. As stated before, these images present a well-defined bimodal histogram, which a priori allows to binarize them very easily by usual threshold procedures. Unfortunately, such direct exploitation leads to erroneous measurements. This can be easily understood by analysing the inherent sources of error which are schematized in Figure 5. Apart from the unavoidable stereological (Fig. 5b) and statistical (Fig. 5d) effects, the major alteration of 




Fig. 5. - Different models for the exploration of the etched surface of a superalloy by a tip and corresponding height-level histograms: a) hypothetic periodic material and infinitely thin tip; b) realistic material and infinitely thin tip; c) realistic material and broad tip; d) realistic material, broad tip and statistical and experimental noise.



Fig. 6. - Transmission electron microscopy image of a $\mathrm{Si}_{3} \mathrm{~N}_{4}$ crystal used as SFM tip probe.

the height-level histogram lies in the fact that the tip size is far to be negligible with regard to the phase scale (Fig. 5c). As illustrated in Figure 6, the $\mathrm{Si}_{3} \mathrm{~N}_{4}$ tip has a pyramidal shape (half-angle $\approx 36^{\circ}$ ) with a rounded vertex of radius ranging between 20 and $50 \mathrm{~nm}$, to be compared with the precipitate size which is about $400 \mathrm{~nm}$. A first way to reduce the measurement bias is therefore to choose the threshold value at the basis of the $\gamma$ peak of the height level histogram, rather than inbetween the two peaks ( $c f$. Fig. 5c) [12]. However, this does not avoid the error due to the impingement of the contributions of the two phases. Different answers to this problem can be proposed, some of them having been already tested.

- From a general point of view, imaging modes which does not require etching of the surface, such as SLAM mode, must be prefered. In that case however, other sources of bias can be effective, which have to be carefully analyzed on the basis of theoretical approaches.

- The tip effect illustrated in Figure 5c can be strictly described as a morphological dilation of the true surface by a non-flat structuring element [19]. Since the shape of this structuring element 


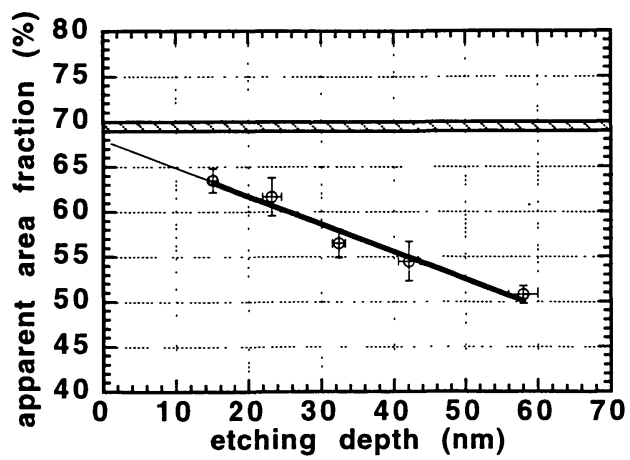

Fig. 7. - Evolution of the apparent $\gamma^{\prime}$ area fraction measured on SFM images, as a function of etching depth measured on the same images. The hatched area indicates the "true" value.

is known ( $c f$. Fig. 6), it is theoretically possible to restore a part of the initial profile by applying the dual transformation, that is a morphological erosion. This has been already tested, not in the case of superalloys, but of ideal and corrugated surfaces [20]. It was concluded that this approach could attenuate, but not completely suppress, the tip-related effects in SFM and STM. The gain will be enhanced if a model of the structure observed is available.

- The importance of bias is directly related to the depth of etching, which is measurable from the distance between the two peaks of the height level histogram. Thus, one solution is to perform measurements after different times of etching, i.e. for different etching depths, then to use a linear extrapolation up to zero etching depth to get the effective value. As illustrated in Figure 7, such procedure allowed to measure a volume fraction of $\gamma^{\prime}$ phase in very good agreement with the one obtained by precipitate extraction and weighing [12]. Apart from the rather long time required for the whole procedure - which in the case of superalloys is comparable with the time required by other quantifying procedures - , one other limitating point is the validity of the linear extrapolation. As it is also possible to use SFM after etching $\gamma$ phase rather than $\gamma^{\prime}[21,22]$, the accuracy would still be enhanced by coupling measurements obtained with the two types of surface preparation.

- The $\gamma-\gamma^{\prime}$ microstructure of single-crystal superalloys can be reasonably modelled by a distribution of perfect cubes of constant size. If also assuming a simple but realistic model for the tip (rounded cone), one can calculate a theoretical height-level histogram. It depends on the tip characteristics (angle of the cone, tip radius), on the etching depth, on the standard deviation of the experimental noise and on the microstructure characteristics (precipitate size and volume fraction). Figure 8 presents two examples of such histograms calculated for two sets of parameters [11]. In return, a given experimental histogram can be fitted to get an estimation of the microstructural parameters. This estimation will be more accurate if some of the parameters of the model can be measured independently. Unlike previous approaches, this latter one is of course limitated to single-crystal superalloys or similar structures. An other microstructural model would be required to treat any other type of material.

\section{Conclusion}

Scanning force microscopy appears to be suitable not only for imaging, but also for quantitatively analysing the microstructure of single-crystal nickel-based superalloys. In particular, it can provide images of the topography of polished/etched surfaces whose resolution and contrast 

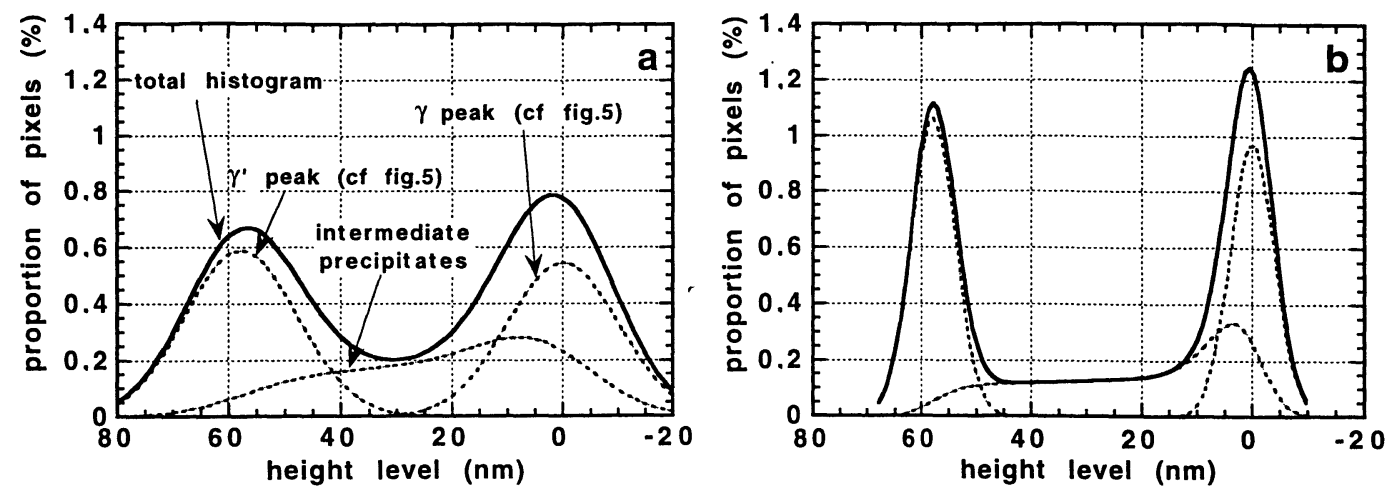

Fig. 8. - Theoretical depth-level histograms calculated for two different sets of modelling parameters (see Ref. [11] for details).

are comparable or even better than those of images from scanning electron microscopy. This enables therefore objective threshold and reproducible measurements. Nevertheless, as any imaging technique dealing with the sub-micronic scale, SFM is subjected to image alterations which, in that case, are mostly due to the non-negligible size of the tip probe. However, the main advantage is that the "transfer function" between the real surface and the final image is relatively easy to estimate, as soon as the geometry of the tip is known. This enables to take into account the inherent analysis bias. Another limitating aspect is the time required for image acquisition, although important improvements have been done -and can still be done- in that field (a $512 \times 512$ SFM image can now be obtained in 1 or $2 \mathrm{mn}$ ). It can be noted that in the case of nickel-based superalloys, the techniques providing accurate absolute values of the pertinent microstructure parameters (precipitate extraction and weighting, atom probe analysis, small angle neutron diffusion, etc...) are also time-consuming.

Our works concerning the enhancement of SFM imaging dealt with single-crystal nickel-based superalloys because there is a real need of fiable measurements for these alloys. Moreover, their microstructure is sufficiently simple to be modelled in a realistic way. Obviously, the potential field of application of SFM in materials science is much wider than superalloys. In terms of microstructure imaging and quantitative analysis, it has a place to take between scanning electron microcopy, which enables rapid observation but can be impeded by complex imaging artefacts, and transmission electron microscopy, which provides relatively fiable images at very fine scales but requires long sample preparation procedures to observe very limitated volumes.

Lastly, SFM used in "SLAM" working mode allows not only to image metallic phases with different elastic properties, but also to provide an estimation of their Young moduli. We believe that this opens a wide range of applications for this technique in materials science, although theoretical and experimental works have to be done to found it more firmly. 


\section{References}

[1] Wiesendanger R., Scanning Probe Microscopy and Spectroscopy - Methods and Applications (Cambridge University Press, 1994).

[2] Sims C.T. and Hagel W.C., The Superalloys (John Wiley \& Sons, New-York, 1972).

[3] Lacaze J. and Hazotte A., Text. Microstr. 13 (1990) 1.

[4] Hazotte A. and Lacaze J., Rev. Mét. - CIT 91 (1994) 277.

[5] Georget D. and Peyroutou C., Proc. of the Eur. Conf. on "High Temperature Materials for Power Engineering 1990", Liège, BELGIUM, sept. 24-27, 1990, Bachelet et al. (Kluwer Academic Pub.) p. 1377

[6] Binnig G., Rohrer H., Gerber C. and Weibel E., Phys. Rev. Lett. 49 (1982) 57.

[7] Binnig G., Quate C.F. and Gerber C., Phys. Rev. Lett. 56 (1986) 930.

[8] Troyon M., Hazotte A., Lei H.N., Bourhettar A. and Perchet A., Proc. of the 10th Eur. Cong. on Electron Microscopy (EUREM 92), Granada, SPAIN, sept. 7-11, 1992, Electron Microscopy 1 (1992) 581.

[9] Bourhettar A., Thèse de Doctorat de l'Université de Reims, France (1994).

[10] Bourhettar A., Hazotte A. and Troyon M., Proc. of the 33rd Col. of the Société Française de Microscopie Électronique (SFME), Lyon, France (1993).

[11] Troyon M., Hazotte A. and Bourhettar A., Micros. Microanal. Microstruct. 5 (1994) 489.

[12] Bourhettar A., Hazotte A. and Troyon M., Mater. Charact. 34 (1995) 265.

[13] Troyon M., Wang Z., Lei H.N., Shang G. and Wagner D., Spectra Analyse 185 (1995) 55.

[14] Troyon M., Lei H.N., Wang Z. and Shang G., Scan. Microsc. Int., to be published.

[15] Caron P. and Khan T., Proc. EUROMAT'89 (Aachen 1989), H.E. Exner, V. Schumacher, Eds. (DGM Informationgessellschaft, Oberursel, Germany, 1990) pp. 333-338.

[16] Burham N.A., Kulik A.J., Gremaud G., Gallo P.J. and Oulevey F., J. Vac. Sci. Technol. B 14 (1996) 794.

[17] Radmacher M., Tillmann R.W. and Gaub H.E., Biophys. J. 64 (1993) 735.

[18] Overney R., Meyer E., Frommer J. and Güntherodt H.J., Langmuir 10 (1994) 1281.

[19] Coster M. and Chermant J.L., Précis d'Analyse d'Images (Éditions du CNRS, 1985).

[20] Bonnet N., Dongmo S., Vautrot P. and Troyon M., Microsc. Microanal. Microstruct. 5 (1994) 477.

[21] Göken M., Fortschr.-Ber. VDI Reihe 18 Nr. 175, Düsseldorf: VDI-Verlag (1995).

[22] Göken M. and Vehoff H., Scr. Mater. 35 (1996) 983. 